\title{
Cerebral Venous Thrombosis: An Important Cause of Bilateral Thalamic Involvement
}

\section{Aravind G' ${ }^{1}$, Monali Chaturvedi ${ }^{2}$, Suman Kushwaha ${ }^{1}$, Aldrin Anthony ${ }^{1}$ and Siddharth Maheshwari ${ }^{1 *}$}

${ }^{1}$ Assistant Professor, Department of Neurology, Institute of Human Behavior and Allied

Sciences, New Delhi, India

${ }^{2}$ Assistant Professor, Department of Neuroradiology, Institute of Human Behavior and

Allied Sciences, New Delhi, India

*Corresponding Author: Siddharth Maheshwari, Assistant Professor, Department of Neurology, Institute of Human Behavior and Allied Sciences, New Delhi, India
Received: May 24, 2021

Published: June 29, 2021

(C) All rights are reserved by Siddharth

Maheshwari., et al.

\begin{abstract}
Cerebral venous thrombosis (CVT) has protean manifestations, radiological features and treatment outcomes. The first case that was described in literature was a 45year old man with headache, seizures and delirium. In India the first case series was reported in 1957. At that time it was a lethal disease with no antemortem diagnostic modalities. With current advances in imaging, it has now become easier to diagnose and treat. We present an interesting case of a 43 year-old male with hemorrhagic infarcts in bilateral basal ganglia and bilateral thalami.

Keywords: Cerebral Venous Thrombosis; Anticoagulant; Bilateral Thalamic Infarcts
\end{abstract}

\section{Introduction}

Cerebral venous thrombosis (CVT) is an uncommon cause of stroke with extremely varied clinical presentations, predisposing factors, imaging findings, and outcomes. CVT was first described in the French literature in 1825, in a 45 year old man who died after a 6 month history of severe headache, epilepsy, and delirium. In 1957 , it was for the first time reported from India in a case series. It was at that time recognized as a diagnosis which was usually made at autopsy and was usually lethal. With more advanced imaging techniques and increased awareness of the disease, the incidence has increased and the prognosis has improved.Bilateral thalamic involvement resembling many diseases in neuroimaging. We present a case report of 43 year-old male with thrombosis of straight and transverse sinus and hemorrhagic infarcts in bilateral basal ganglia and bilateral thalami. Bilateral symmetrical hyper intensities in basal ganglia and thalami on neuroimaging may be due to various causes of diverse etiology and CVT remains an important cause. Early, accurate and prompt diagnosis is crucial as appropriate therapy can reverse the disease process and significantly reduce the risk of acute complications and long term sequelae.

\section{Case History}

43 year old male, K/C/O chronic alcoholism with dependence, DVT Rt lower limb and hypertension. He was on oral anticoagulant(dabigatran) with poor compliance.

He presented with c/o severe headache for one week and altered sensorium for three days. Headache was severe in intensity, holocranial, not relieved by medications. There was no history of nausea, vomiting, decreased vision, seizure or fever.

$\mathrm{H} / \mathrm{O}$ altered sensorium for three days in the form of drowsiness, irrelevant talking, not recognizing distant relatives and increased sleep. There was no history of urinary or bowel incontinence.

Patient was admitted with above complaints in our hospital and evaluated. Examination s/o normal vitals, disorientation to time, place and person, postural and intention tremor but no flapping tremor.

Ct head done which was s/o microhaemorrhages in periventricular region and dense straight sinus. Blood investigation including 
LFTs, PT/INR and serum ammonia level were normal. Coagulation profile done - negative.

MRI brain done which was s/o T2/FLAIR hyperintensity in b/l thalamus, basal ganglia, external capsule and splenium of corpus callosum with diffusion restriction and blooming on GRE s/o haemorrhage. These findings were s/o venous infarct.

MRV brain done which was s/o thrombus in straight sinus and draining veins.

Diagnosis of cerebral venous thrombosis (deep venous system) was made and he was treated with anticoagulants, thiamine, symptomatic treatment and iv fluids. He improved significantly with treatment and discharged in stable condition.

\section{Discussion}

The first description of CVT, appearing in the French literature in 1825 , was by Ribes, in a 45 year old man who died after a 6 month history of severe headache, epilepsy, and delirium [1]. In 1957, Padmavati., et al. for the first time from India, reported 15 cases of CVT in puerperium in an epidemiological study evaluating the causes of hemiplegia in 44 women [2].

CVT is an uncommon cause of stroke which affects almost 3-4 adults per million population annually [3]. Although, there are no population based studies from India estimating incidence of CVT, various hospital based studies have found that CVT comprises $10 \%$ of all stroke cases in our country [4]. Superior sagittal sinus is the most commonly affected sinus followed by transverse and sigmoid sinus.

DCVT term is used for thrombosis of internal cerebral vein, vein of Galen, basal vein of Rosenthal and its tributaries, and is seen in $10 \%$ cases of CVT [5].

Inherited thrombophilic states, hyperhomocystinemia, drugs, pregnancy, dehydration, malignancy and autoimmune conditions are the usual causes. However, in 15\% cases of CVT, no predisposing factor could be found [6]. Headache is the most common symptom and is seen in almost 70\%-90\% cases [7]. Hemiparesis, seizures, loss of consciousness, papilloedema and visual disturbances are other commonly observed presentation in CVT. The clinical presentation of DCVT is non specific and patient can present with short history of altered sensorium, aphasia, seizures, nausea, vomiting, coma or death [8]. CT finding in DCVT often shows hypoattenuation of bilateral thalami and basal ganglia. However, the venous system which includes vein of Galen, vein of Rosenthal, internal cerebral vein and straight sinus appear hyperattenuated on CT [9].

The most sensitive imaging technique for early diagnosis of DCVT is MRI with MRV [10]. The deep venous system drains thalamus, basal ganglia, corpus callosum, inferior frontal lobes and deep white matter of parietal and temporal lobe [11]. So, thrombosis of deep cerebral vein causes vasogenic oedema and ischemic changes in these area, which appears hyperintense on T2-weighted images and hypointense onT1-weighted images.

MRI involvement of bilateral thalamus is seen in various other conditions like JE, Wilson's disease, Wernicke's encephalopathy, hypoxic-ischemic encephalopathy and Multiple Sclerosis [12]. MRI finding of JE closely resembles that of DCVT and shows hyperintense lesion in bilateral thalami on T2-W images, while hemorrhagic transformation appears hyperintense on T1-W image. The oedema is predominantly cytotoxic as compared to vasogenic edema in DCVT. MRI imaging often shows thrombus in deep cerebral vein in case of DCVT which can be confirmed further by MRV. MRV shows abnormal signal intensity in corresponding sinus with absence of flow [10].

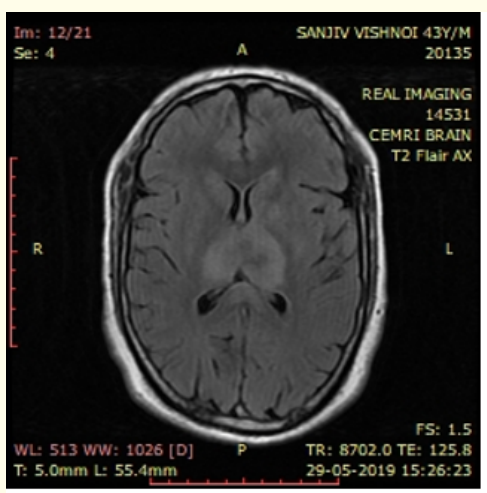

(A) 


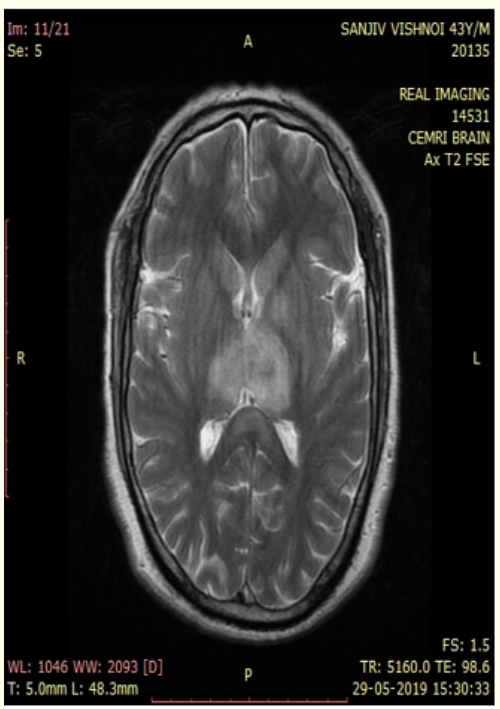

(B)

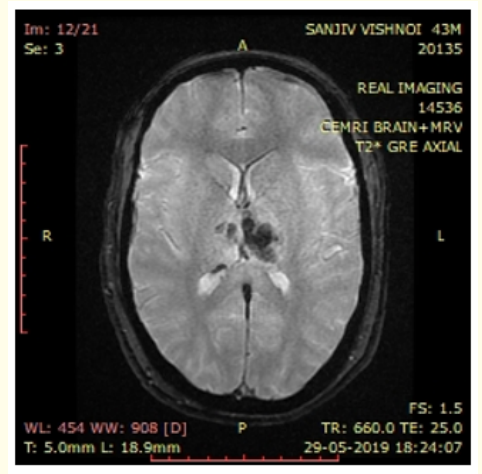

(C)

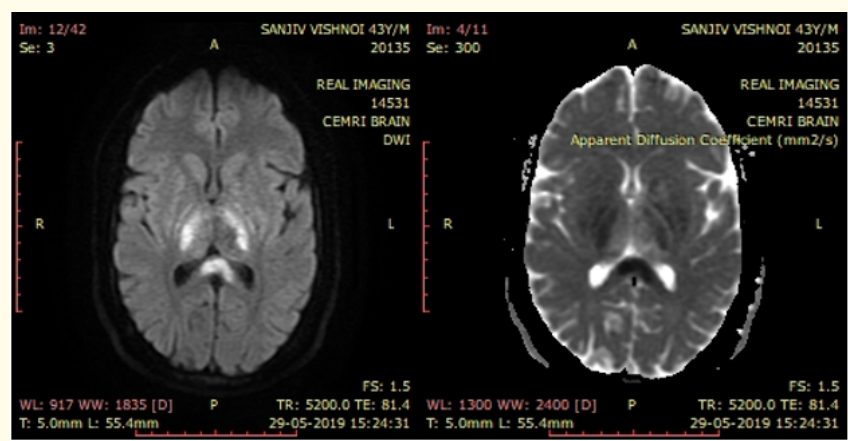

(D, E)

Figure 1: A and B: T2 and FLAIR hyperintensites in bilateral thalami and splenium of corpus callosum. C: GRE images seen in bilateral thalami and straight sinus suggestive of bleed with thrombus in straight sinus. D, E: DWI and ADC images showing restricted diffusion in bilateral thalami and splenium of corpus callosum.

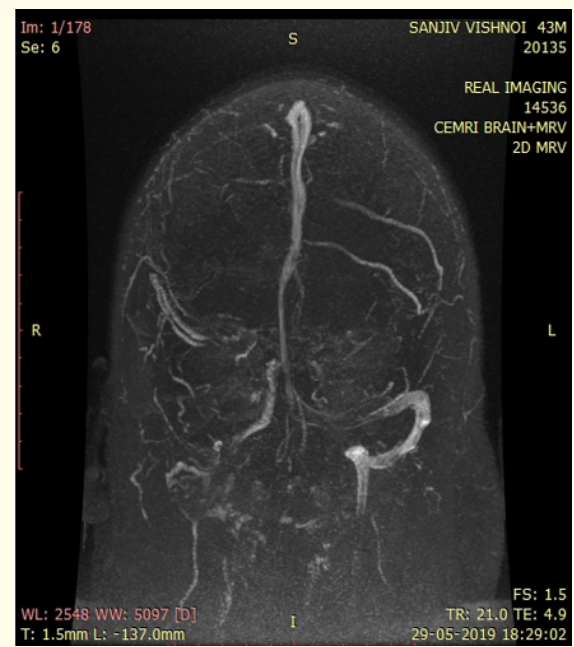

Figure 2: MR venography showing thrombus in straight sinus extending to torcula and bilateral transverse sinuses.

\section{Conclusion}

A high index of suspicion is needed for early diagnosis of DCVT. Bilateral symmetrical hyper intensities in basal ganglia and thalami on neuroimaging may be due to various causes of diverse etiology and CVT remains an important cause. It has favourable outcome, if recognized and treated early. MRI brain should be done early in cases of unexplained altered sensorium. If MRI is suggestive of DCVT, then MRV should be done for confirmation. Any reversible cause of thrombosis should be treated.

\section{Bibliography}

1. Ribes MF. Des recherches faites sur la phlebite Revue Medical Francais et Etrangere er Journal de clinique del 'Hotel Dieu et de la Charite de Paris 3 (1825): 5.

2. Padmavati S., et al. "A clinical study of 44 cases of hemiplegia in adult women". Neurology India 5 (1957): 59-65.

3. Stam J. "Thrombosis of the cerebral veins and sinuses". The New England Journal of Medicine 352.17 (2005): 1791-1798.

4. Banerjee AK., et al. "Cerebrovascular disease in north-west India: a study of necropsy material". Journal of Neurology, Neurosurgery, and Psychiatry 52 (1989): 512-515. 
5. Ferro JM., et al. "ISCVT Investigators: prognosis of cerebral vein and dural sinus thrombosis: results of the international study on cerebral vein and dural sinus thrombosis (ISCVT)". Stroke 35 (2004): 664-670.

6. Schaller B and Graf R. "Cerebral venous infarction: the pathophysiological concept". Cerebrovascular Disease 18.3 (2004): 179-188.

7. Ameri A and Bousser MG. "Cerebral venous thrombosis". Neurologic Clinics 10.1 (1992): 87-111.

8. Crawford SC., et al. "Thrombosis of deep venous drainage of the brain in adults: analysis of seven cases with review of the literature". Archives of Neurology 52 (1995): 1101-1108.

9. Eick JJ., et al. "Computed tomography of deep cerebral venous thrombosis in children". Radiology 140 (1981): 399-402.

10. Ayanzen RH., et al. "Cerebral MR venography: normal anatomy and potential diagnostic pitfalls". American Journal of Neuroradiology 21.1 (2000): 74-78.

11. Meder JF., et al. "Venous territories of the brain". Journal of Neuroradiology 21.2 (1994): 118-133.

12. Kalita J and Misra UK. "Comparison of CT and MRI findings in the diagnosis of Japanese encephalitis". Journal of Neurology Science 174.1 (2000): 3-8.

\section{Volume 4 Issue 7 July 2021}

(C) All rights are reserved by Siddharth Maheshwari., et

al. 